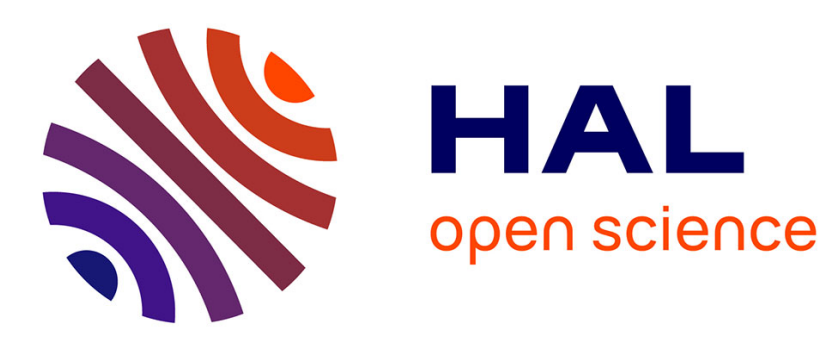

\title{
Control of nonlinear and LPV systems: interval observer-based framework
}

Denis Efimov, Tarek Raïssi, Ali Zolghadri

\section{To cite this version:}

Denis Efimov, Tarek Raïssi, Ali Zolghadri. Control of nonlinear and LPV systems: interval observer-based framework. IEEE Transactions on Automatic Control, 2013, 58 (3), pp.773-778. 10.1109/TAC.2013.2241476 . hal-00664106

\section{HAL Id: hal-00664106 https://hal.science/hal-00664106}

Submitted on 21 Nov 2012

HAL is a multi-disciplinary open access archive for the deposit and dissemination of scientific research documents, whether they are published or not. The documents may come from teaching and research institutions in France or abroad, or from public or private research centers.
L'archive ouverte pluridisciplinaire HAL, est destinée au dépôt et à la diffusion de documents scientifiques de niveau recherche, publiés ou non, émanant des établissements d'enseignement et de recherche français ou étrangers, des laboratoires publics ou privés. 


\title{
Control of nonlinear and LPV systems: interval observer-based framework
}

\author{
Denis Efimov, Tarek Raïssi, and Ali Zolghadri
}

\begin{abstract}
The problem of output stabilization of a class of nonlinear systems subject to parametric and signal uncertainties is studied. First, an interval observer is designed estimating the set of admissible values for the state. Next, it is proposed to design a control algorithm for the interval observer providing convergence of interval variables to zero, that implies a similar convergence of the state for the original nonlinear system. An application of the proposed technique shows that a robust stabilization can be performed for linear time-varying and LinearParameter-Varying (LPV) systems without assumption that the vector of scheduling parameters is available for measurements. Efficiency of the proposed approach is demonstrated through two examples.
\end{abstract}

Index Terms-Nonlinear stabilization, LPV systems and control, interval estimation

\section{INTRODUCTION}

The nonlinear system stabilization has been an area of active research during the last two decades [2], [9], [11]. Most of the proposed approaches appeal to particular structural characteristics of a given class of nonlinear systems. Frequently a partial similarity to linear systems is used to take advantage of the well established solutions for observer or control design. For example, the class of Lipschitz nonlinear systems forms a subclass of nonlinear ones, which can be estimated and controlled applying linear control approaches [13], [17]. This class of nonlinear systems is considered in the paper under the assumption that the model is subject to uncertain time-varying parameters. The proposed methodology ensures stabilization for all parameter values belonging to a given interval.

An important framework, which has been largely investigated to solve the problems of estimation and control for generic nonlinear systems, is based on LPV transformations [14], [22]. There exist several approaches to equivalently represent a nonlinear system in a LPV form [6], [15], [24]. It is worth to note that such a procedure is not based on approximate linearization. It is global and it transforms the nonlinear system by introducing extended parametric uncertainties to the LPV setting. There are several methods for estimation of LPV systems, one of them is based on design of interval state observers [5], [16], [20], [19], which provide two variables evaluating the lower and upper bounds for state values of LPV

The first author is with the Non-A project at INRIA - LNE, Parc Scientifique de la Haute Borne, 40 avenue Halley, Bât.A Park Plaza, 59650 Villeneuve d'Ascq, France, denis.efimov@inria.fr. The second author is with Conservatoire National des Arts et Métiers (CNAM), Département EASY, Cedric - laetitia 292, Rue St-Martin, 75141 Paris, France, tarek.raissi@cnam.fr. The last author is with University of Bordeaux, IMS-lab, Automatic control group, 351 cours de la libération, 33405 Talence, France, Ali.Zolghadri@imsbordeaux.fr. systems in real time. Control of LPV systems is more challenging and has been intensively studied [3], [4], [10], [21]. Classically, the vector of scheduling parameters is assumed to be measured (at least partly or with an error) [1], [7], [12]. However, in some cases, this assumption may become hard to satisfy because some relevant physical parameters that can be served as scheduling parameters are not measured or their measurements are not judged reliable. For example, to generate a LPV model for a nonlinear aircraft, usually mass and center of gravity are used as scheduling parameters. Although these parameters are measured and available on-board (for example mass estimation based on fuel consumption), their measurements are relatively crude and they should be considered to be an interval, rather than a single point measurement.

In the present work the classical assumption, that the vector of scheduling parameters is available for measurements, is dropped. The proposed dynamic output feedback approach is based on an interval state observer design for a given class of nonlinear uncertain systems. An interval observer was used for a bioreactor control in [18], where the interval estimates were substituted in the control law to ensure the state convergence to a steady state. In the present work this idea is generalized, and the control is designed to stabilize the interval observer ensuring convergence to a vicinity of zero for the bounding variables. Since the computed bounds of the state have to be valid for any control, the plant state vector also converges to the origin. An advantage of the proposed approach is that it allows us to stabilize a wide spectrum of nonlinear uncertain systems with partial measurements.

The paper is organized as follows. Some preliminaries are given in Section 2. The problem statement and the system equations are given in Section 3. The interval observer design is presented in Section 4. Section 5 is devoted to the control design. Finally, two simulation examples are given in Section 6 to demonstrate the efficiency of the developed technique.

\section{PRELIMINARIES}

In this work for any two vectors $x_{1}, x_{2}$ or matrices $A_{1}, A_{2}$ the relations $x_{1} \leq x_{2}, x_{1} \geq x_{2}, A_{1} \leq A_{2}, A_{1} \geq A_{2}$ are understood elementwise (as a collection of inequalities satisfied for all elements). A square and symmetric positive (semi)definite matrix $P$ is defined by $P \succ 0(P \succeq 0)$, the inverse symbols $\prec, \preceq$ are stated for negative (semi)definite matrices. The symbol $|\cdot|$ is used to denote vector or corresponding induced matrix norms. The symbol $I$ denotes the identity matrix, $\lambda_{\min }(A)$ and $\lambda_{\max }(A)$ are respectively stated for the minimal and maximal eigenvalues of a symmetric matrix $A$. The sequence of integers $1, \ldots, n$ is denoted as $\overline{1, n}$. 
Recall that a square matrix $S$ with dimension $n \times n$ is called Metzler if all its off-diagonal elements are nonnegative: $S_{i, j} \geq$ $0,1 \leq i \neq j \leq n$. For such Metzler matrix $S$, the system

$$
\dot{z}=S z+r(t), z \in \mathbb{R}^{n}, r: \mathbb{R}_{+} \rightarrow \mathbb{R}_{+}^{n}
$$

is monotone (cooperative) [23] and has nonnegative solutions, i.e. if $z(0) \geq 0$ then $z(t) \geq 0$ for all $t \geq 0$.

\section{PROBLEM STATEMENT}

Consider the nonlinear system

$$
\dot{x}=A x+B(\theta(t)) u+f(x, y, \theta(t)), y=C x,
$$

where $x \in \mathbb{R}^{n}, y \in \mathbb{R}^{p}, u \in \mathbb{R}^{m}$ are the state, the output and the control respectively, $\theta \in \Theta \subset \mathbb{R}^{q}$ is the vector of uncertain possibly time-varying parameters, the set $\Theta$ is assumed to be given, $t \geq 0$. The constant matrices $A$ and $C$ and the nonlinear functions $B: \mathbb{R}^{q} \rightarrow \mathbb{R}^{n \times m}, f: \mathbb{R}^{n+p+q} \rightarrow \mathbb{R}^{n}$ are known, the function $f$ ensures uniqueness and existence of the system solutions at least locally. Without any loss of generality, assume that $f(0,0, \theta)=0$ for any $\theta \in \Theta$.

The goal of the paper is to design a dynamic output feedback ensuring the system (1) (practical) stabilization at the origin. The forthcoming investigation is based on the following properties of the system (1):

There are functions $f, \bar{f}: \mathbb{R}^{2 n+p} \rightarrow \mathbb{R}^{n}$ and matrices $B_{\min }, B_{\max }$ such that the relations

$$
\begin{gathered}
\underline{f}(\underline{x}, \bar{x}, y) \leq f(x, y, \theta) \leq \bar{f}(\underline{x}, \bar{x}, y), \\
B_{\min } \leq B(\theta) \leq B_{\max }
\end{gathered}
$$

are satisfied provided that $\underline{x} \leq x \leq \bar{x}$ and $\theta \in \Theta$. The functions $f, \bar{f}$ can be computed under assumption that $\underline{\theta} \leq \theta \leq \bar{\theta}$ for all $\theta \in \Theta$ and some $\underline{\theta}, \bar{\theta} \in \mathbb{R}^{q}$. Note that under assumption (A1) for any $u \in \mathbb{R}^{m}$ the inequalities

$$
\underline{B}(u) u \leq B(\theta) u \leq \bar{B}(u) u
$$

are satisfied for

$\underline{B}^{(i)}(u)=\left\{\begin{array}{l}B_{\min }^{(i)} \text { if } u_{i} \geq 0 ; \\ B_{\max }^{(i)} \text { if } u_{i}<0,\end{array} \quad \bar{B}^{(i)}(u)=\left\{\begin{array}{c}B_{\max }^{(i)} \text { if } u_{i} \geq 0 ; \\ B_{\min }^{(i)} \text { if } u_{i}<0,\end{array}\right.\right.$

where the upper index $i$ for a matrix $B^{(i)}$ denotes the $i$-th column of the matrix, and the lower index $i$ for a vector $u_{i}$ returns the $i$-th element of the vector.

In this paper the vector $\theta$ may play a role of the vector of scheduling parameters in the LPV representation:

$$
\dot{x}=A(\theta(t)) x+B(\theta(t)) u
$$

or simply a parameter vector of (1). The system (2) can be presented in the form (1) with $f(x, y, \theta)=[A(\theta)-A] x$. The control design will be presented for more generic nonlinear system (1), the system (2) will be used as a special case. Note that assumption (A1) is satisfied for the LPV system (2) if, for example, there exists a matrix $\Delta A \geq 0$ such that

$$
A-\Delta A \leq A(\theta) \leq A+\Delta A,
$$

for any $\theta \in \Theta$, in this case $\underline{f}(\underline{x}, \bar{x}, y)=-\Delta A(\bar{x}-\underline{x})$, $\bar{f}(\underline{x}, \bar{x}, y)=\Delta A(\bar{x}-\underline{x})$.

\section{INTERVAL STATE OBSERVER}

In this section based on assumption (A1) we are going to design an interval observer for (1) using the results from [5], [20]. To simplify the presentation we introduce an auxiliary assumption (weaker than the assumptions in [5], [20]).

There exists a matrix $L$ such that the matrix $A-$ $L C$ is Metzler.

Note that we do not require here any stability properties for the matrix $A-L C$. In this case, an interval observer for the system (1) can be written as follows [5], [20]:

$$
\begin{aligned}
& \dot{\dot{x}}=A \underline{x}+\underline{B}(u) u+f(\underline{x}, \bar{x}, y)+L(y-C \underline{x}), \\
& \dot{\bar{x}}=A \bar{x}+\bar{B}(u) u+\overline{\bar{f}}(\underline{x}, \bar{x}, y)+L(y-C \bar{x}) .
\end{aligned}
$$

In this paragraph we implicitly assume that the control $u$ does not violate the conditions of solutions existence into the system (3), and that the system (3) solutions are defined for all $t \geq 0$.

Theorem 1. Let assumptions (A1),(A2) and the constraint $\underline{x}(0) \leq x(0) \leq \bar{x}(0)$ be satisfied, then for any control $u$ the solutions of the system (1), (3) satisfy:

$$
\underline{x}(t) \leq x(t) \leq \bar{x}(t), \forall t \geq 0 .
$$

Proof: Introducing the estimation errors $\underline{e}=x-\underline{x}$ and $\bar{e}=\bar{x}-x$ from (1) and (3) we get that

$$
\begin{aligned}
\underline{\dot{e}} & =(A-L C) \underline{e}+\underline{d}(t), \dot{\bar{e}}=(A-L C) \bar{e}+\bar{d}(t), \\
\underline{d} & =[B(\theta)-\underline{B}(u)] u+[f(x, y, \theta)-\underline{f}(\underline{x}, \bar{x}, y)], \\
\bar{d} & =[\bar{B}(u)-B(\theta)] u+[\bar{f}(\underline{x}, \bar{x}, y)-f(x, y, \theta)] .
\end{aligned}
$$

By (A1) we have $\underline{e}(0) \geq 0, \bar{e}(0) \geq 0$ and $\underline{d}(0) \geq 0, \bar{d}(0) \geq 0$. From assumption (A2) the dynamics of the estimation errors is cooperative. Therefore, $\underline{e}(t) \geq 0, \bar{e}(t) \geq 0$ for all $t \geq 0$, that is necessary to prove.

The theorem does not claim that variables $\underline{x}(t)$ and $\bar{x}(t)$ are bounded, it establishes the order relations only for any control $u$. Due to nonlinear nature of the plant and coupling among the systems (1), (3), even Hurwitz property of the matrix $A-$ $L C$ (usual assumption for interval observers [5], [20]) does not ensure boundedness of the solutions $\underline{x}(t)$ and $\bar{x}(t)$. The solution $x(t)$ also can be unbounded. To ensure the overall boundedness of solutions for the systems (1), (3) we have to design a stabilizing control algorithm.

Remark 1. Before we proceed with the control design, it is worth to note that the assumption (A2) can be relaxed. Actually the existence of a nonsingular matrix $T$ is needed such that the matrix $S=T^{-1}(A-L C) T$ is Metzler [19]. Indeed, introducing new variables $x=T z$, the system (1) can be presented as follows:

$$
\begin{gathered}
\dot{z}=T^{-1} A T z+T^{-1} B(\theta(t)) u+T^{-1} f(T z, y, \theta(t)), \\
y=C T z .
\end{gathered}
$$

Obviously, if the assumption (A1) is satisfied for the system (1), then a similar property holds for the system (4) with the functions $T^{-1} B(\theta(t))$ and $T^{-1} f(T z, y, \theta(t))$. Indeed, in this case under assumption (A1) we have:

$$
\begin{gathered}
\underline{f}^{z}(\underline{z}, \bar{z}, y)=\bar{T}_{i} \underline{\varphi}(\underline{z}, \bar{z}, y)-\underline{T}_{i} \bar{\varphi}(\underline{z}, \bar{z}, y) \\
\leq T^{-\underline{1} f}(T z, y, \theta(t)) \\
\leq \bar{T}_{i} \bar{\varphi}(\underline{z}, \bar{z}, y)-\underline{T}_{i} \underline{\varphi}(\underline{z}, \bar{z}, y)=\bar{f}^{z}(\underline{z}, \bar{z}, y),
\end{gathered}
$$




$$
\begin{gathered}
\underline{B}^{z}(u) u=\left[\bar{T}_{i} \underline{B}(u)-\underline{T}_{i} \bar{B}(u)\right] u \leq T^{-1} B(\theta) u \\
\leq\left[\bar{T}_{i} \bar{B}(u)-\underline{T}_{i} \underline{B}(u)\right] u=\bar{B}^{z}(u) u, \\
\underline{\varphi}(\underline{z}, \bar{z}, y)=f(\bar{T} z-\underline{\underline{z}} \bar{z}, \bar{T} \bar{z}-\underline{T z}, y), \\
\bar{\varphi}(\underline{z}, \bar{z}, y)=\overline{\bar{f}}(\bar{T} \underline{z}-\underline{T} \bar{z}, \bar{T} \bar{z}-\underline{T z}, y)
\end{gathered}
$$

and $\bar{T}=\max \{0, T\}, \underline{T}=\bar{T}-T, \bar{T}_{i}=\max \left\{0, T^{-1}\right\}, \underline{T}_{i}=$ $\bar{T}_{i}-T^{-1}$. Then, the interval observer (3) can be written for the system (4) as follows:

$$
\begin{aligned}
& \dot{\dot{z}}=S \underline{z}+\underline{B}^{z}(u) u+\underline{f}^{z}(\underline{z}, \bar{z}, y)+T^{-1} L y, \\
& \dot{\bar{z}}=S \bar{z}+\bar{B}^{z}(u) u+\overline{\bar{f}}^{z}(\underline{z}, \bar{z}, y)+T^{-1} L y .
\end{aligned}
$$

In the new coordinates, the observer (5) is similar to (3). By introducing the estimation errors $\underline{e}=z-\underline{z}$ and $\bar{e}=\bar{z}-z$ it is possible to show that their dynamics are cooperative and

$$
\underline{z}(t) \leq z(t) \leq \bar{z}(t), \forall t \geq 0
$$

provided that $\underline{z}(0) \leq z(0) \leq \bar{z}(0)$. Therefore, for brevity of presentation all results below are stated for the case of assumption (A2), taking in mind the possibility of its relaxation.

Remark 2. Another interval observer is proposed in [16], for (1) under assumption (A1) it can be written as follows

$$
\begin{aligned}
& \dot{\dot{x}}=A^{1} \underline{x}+A^{2} \bar{x}+\underline{B}(u) u+\underline{f}(\underline{x}, \bar{x}, y), \\
& \dot{\bar{x}}=A^{1} \bar{x}+A^{2} \underline{x}+\overline{\bar{B}}(u) u+\overline{\bar{f}}(\underline{x}, \bar{x}, y),
\end{aligned}
$$

where $A_{i, i}^{1}=A_{i, i}, A_{i, j}^{1}=\max \left\{0, A_{i, j}\right\}$ for $i=\overline{1, n}, j=\overline{1, n}$, $j \neq i$ and $A^{2}=A-A^{1}$. The interval observer (6) has not the gain $L$, thus it is independent on assumption (A2). If $\underline{f}$ and $\bar{f}$ are independent on $y$, then the observer is an autonomous system. This observer under the constraint $\underline{x}(0) \leq x(0) \leq \bar{x}(0)$ for any control $u$ ensures that $\underline{x}(t) \leq x(t) \leq \bar{x}(t), \forall t \geq 0$. The control approach proposed below can also be applied to (6). This possible extension is omitted for brevity of presentation.

\section{Control Design}

This section contains the main contribution of this work, which consists in solving the stabilization problem for the completely known system (3) instead of (1). Under conditions of Theorem 1 , if both $\underline{x}(t)$ and $\bar{x}(t)$ converge to zero, then the state $x(t)$ also has to converge to zero, and boundedness of $x(t)$ follows by the same property of $\underline{x}(t)$ and $\bar{x}(t)$. In this case the signal $y(t)$ is treated in the system (3) as a state dependent disturbance with an upper bound

$$
|y(t)| \leq|C|(|\underline{x}(t)|+|\bar{x}(t)|), \forall t \geq 0 .
$$

Therefore, it is required to stabilize the system (3) uniformly (or robustly) with respect to the input $y$. Applying the same arguments in the case of Remark 1, the system (1) stabilization follows by the interval observer stabilization in coordinates $\underline{z}, \bar{z}$ (the matrix $T$ is nonsingular). In the case of Remark 2, stabilization of (1) follows by the system (6) stabilization.

The advantages of such reduction are that the system (3) is completely known and the state vector $\underline{x}(t), \bar{x}(t)$ is available. However, the dimension of (3) is twice bigger than the corresponding dimension of the system (1) while the control vector $u$ preserves its size. Another difficulty is that the system (3) has variable structure if the matrix functions $\underline{B}$ and $\bar{B}$ depend on $u$ or if the matrix function $B$ in (1) depends on $\theta$ (if this is not the case and $B(\theta)=B$, then $\underline{B}(u)=\bar{B}(u)=B$ ).

The nonlinearity of the system (3) inherited from (1) represents an obstacle, since there is no common approach to robustly stabilize a generic nonlinear system. From another side, there exist several techniques obtained for special classes of nonlinear systems [9], [11]. For this purpose some restrictions on the functions $\underline{f}$ and $\bar{f}$ are introduced.

\section{A. Lipschitz case}

Let there exist constants $\bar{a}_{i} \geq 0, \underline{a}_{i} \geq 0, i=$ $\overline{1,4}$ such that for any $\bar{x}, \underline{x} \in \mathbb{R}^{n}, y \in \mathbb{R}^{p}$ the inequalities are satisfied:

$$
\begin{aligned}
& |\bar{f}(\underline{x}, \bar{x}, y)| \leq \bar{a}_{1}|\bar{x}|+\bar{a}_{2}|\underline{x}|+\bar{a}_{3}|y|+\bar{a}_{4}, \\
& |\underline{f}(\underline{x}, \bar{x}, y)| \leq \underline{a}_{1}|\bar{x}|+\underline{a}_{2}|\underline{x}|+\underline{a}_{3}|y|+\underline{a}_{4} .
\end{aligned}
$$

The introduced assumption deals with the known functions $f$ and $\bar{f}$ instead of the uncertain $f$ in the plant equations (1). If stabilization is required for a compact predefined set of initial conditions, then for rather wide spectrum of nonlinear functions $f$ the majorant nonlinearities $f$ and $\bar{f}$ can be chosen locally to satisfy the assumption (A3). Indeed, roughly speaking this assumption says that the nonlinearities $f$ and $\bar{f}$ are globally Lipschitz or bounded. The local Lipschitz property is sufficient if stabilization into a predefined set is needed.

Due to Lipschitz property of (3) under assumption (A3), the control is chosen as a conventional state linear feedback:

$$
u=\underline{K x}+\bar{K} \bar{x},
$$

where $\underline{K}$ and $\bar{K}$ are two feedback gains to be designed. Substitution of the control (7) into the equations (3) gives:

$$
\begin{aligned}
& \dot{\dot{x}}=[A-L C+\underline{B}(u) \underline{K}] \underline{x}+\underline{B}(u) \bar{K} \bar{x}+\underline{f}(\underline{x}, \bar{x}, y)+L y, \\
& \dot{\bar{x}}=[A-L C+\bar{B}(u) \overline{\bar{K}}] \bar{x}+\overline{\bar{B}}(u) \underline{K x}+\underline{\bar{f}}(\underline{x}, \bar{x}, y)+L y .
\end{aligned}
$$

The linear part of the system depends on the sign of the control (7) and is defined by the following $2^{m}$ matrices:

$$
G_{k}=\left[\begin{array}{cc}
A-L C+\underline{B}_{k} \underline{K} & \underline{B}_{k} \bar{K} \\
\bar{B}_{k} \underline{K} & A-L C+\bar{B}_{k} \bar{K}
\end{array}\right],
$$

for $k=1, \ldots, 2^{m}$, where $\bar{B}_{k}=B_{\max }+B_{\min }-\underline{B}_{k}$ and $\underline{B}_{k}$ represents all possible realizations of $\underline{B}(u)$ for different unknown signs of the elements of $u$, i.e. $\underline{B}_{k}^{(i)}$ is composed by $B_{\min }^{(i)}$ or $B_{\max }^{(i)}$ for each $i=1, \ldots m$ (there are $2^{m}$ variants of such compositions). Thus the system (8) is nonlinear and it has multiple-mode linear part.

Theorem 2. Let assumptions (A1)-(A3) hold, $\underline{x}(0) \leq x(0) \leq$ $\bar{x}(0)$ and there exist a constant $\epsilon>0$ and matrices $P^{T}=$ $P \succ 0, Q^{T}=Q \succ 0$ and $W$ such that the LMIs

$$
\begin{gathered}
{\left[\begin{array}{cc}
{\left[\epsilon^{2} I+Q\right]^{-1}} & P^{-1} \\
P^{-1} & \Pi_{k}-2(\alpha / \epsilon)^{2} I
\end{array}\right] \succeq 0,} \\
\Pi_{k}=-P^{-1} Z^{T}-Z P^{-1}-W^{T} N_{k}^{T}-N_{k} W, \\
Z=\left[\begin{array}{cc}
A-L C & 0 \\
0 & A-L C
\end{array}\right], N_{k}=\left[\begin{array}{c}
\underline{B}_{k} \\
B_{k}
\end{array}\right]
\end{gathered}
$$

are satisfied for all $k=1, \ldots, 2^{m}$, where $\alpha=2 \max _{s=1,2}\left\{\bar{a}_{s}+\right.$ $\left.\underline{a}_{s}\right\}+2|C|\left(\bar{a}_{3}+\underline{a}_{3}+2|L|\right)$. Then $[\underline{K} \bar{K}]=W P$ and solutions 
of the system (1), (3), (7) admit the estimate for all $t \geq 0$ and $x(0) \in \mathbb{R}^{n}$ :

$$
|x(t)| \leq \kappa(|\underline{x}(0)|+|\bar{x}(0)|) e^{-0.5 \eta t}+\rho \varrho / \epsilon,
$$

where $\eta=\frac{\lambda_{\min }(Q)}{\lambda_{\max }(P)}, \kappa=\sqrt{\frac{\lambda_{\max }(P)}{\lambda_{\min }(P)}}, \rho=\kappa \sqrt{\frac{2 \lambda_{\max }(P)}{\eta}}$ and $\varrho=\bar{a}_{4}+\underline{a}_{4}$.

Proof: Since all conditions of Theorem 1 are satisfied, we have $\underline{x}(t) \leq x(t) \leq \bar{x}(t)$ for all $t \geq 0$ and $|y| \leq 2|C||\xi|$, where $\xi=\left[\underline{x}^{T} \bar{x}^{T}\right]^{T}$ is the state vector of the system (8). If the LMIs are satisfied, then using the Schur complement it is possible to show that the Riccati inequalities

$$
G_{k}^{T} P+P G_{k}+\epsilon^{2} I+2(\alpha / \epsilon)^{2} P^{2}+Q \preceq 0
$$

hold for all $k=1, \ldots, 2^{m}$. Consider for the system (8) the Lyapunov function $V=\xi^{T} P \xi$ :

$$
\dot{V}=\xi^{T}\left(G_{k}^{T} P+P G_{k}\right) \xi+2 \xi^{T} P[F(\xi, y)+\Lambda y],
$$

where $F(\xi, y)=\left[\underline{f}(\underline{x}, \bar{x}, y)^{T} \bar{f}(\underline{x}, \bar{x}, y)^{T}\right]^{T}$ and $\Lambda=$ $\left[\begin{array}{ll}L^{T} & L^{T}\end{array}\right]^{T}$. Owing the previous definitions

$$
\begin{aligned}
|F(\xi, y)| \leq & \left(\bar{a}_{1}+\underline{a}_{1}\right)|\bar{x}|+\left(\bar{a}_{2}+\underline{a}_{2}\right)|\underline{x}| \\
& +\left(\bar{a}_{3}+\underline{a}_{3}\right)|y|+\left(\bar{a}_{4}+\underline{a}_{4}\right) \\
\leq 2 \quad & \max _{s=1,2}\left\{\bar{a}_{s}+\underline{a}_{s}\right\}|\xi| \\
& +\left(\bar{a}_{3}+\underline{a}_{3}\right)|y|+\left(\bar{a}_{4}+\underline{a}_{4}\right) \\
|\Lambda| \leq 2|L|, &
\end{aligned}
$$

and completing squares, we obtain $(|F(\xi, y)+\Lambda y| \leq \alpha|\xi|+\varrho)$ :

$$
\begin{aligned}
\dot{V} \leq & \xi^{T}\left(G_{k}^{T} P+P G_{k}+\epsilon^{2} I\right) \xi \\
& +\epsilon^{-2}[F(\xi, y)+\Lambda y]^{T} P^{2}[F(\xi, y)+\Lambda y] \\
\leq & \xi^{T}\left(G_{k}^{T} P+P G_{k}+2(\alpha / \epsilon)^{2} P^{2}\right. \\
& \left.+\epsilon^{2} I\right) \xi+2(\varrho / \epsilon)^{2}|P|^{2} \\
\leq & -\xi^{T} Q \xi+2(\varrho / \epsilon)^{2}|P|^{2} \\
\leq & -\eta V+2(\varrho / \epsilon)^{2}|P|^{2} .
\end{aligned}
$$

Therefore, the following time estimate is satisfied for $\xi$ :

$$
|\xi(t)| \leq \kappa|\xi(0)| e^{-0.5 \eta t}+\rho \varrho / \epsilon,
$$

it is bounded converging asymptotically to a vicinity of zero (the system (8) is practically stable). Since conditions of Theorem 1 are satisfied we have that $\underline{x}(t) \leq x(t) \leq \bar{x}(t)$ $\forall t \geq 0$, that implies boundedness and convergence of $x(t)$.

The proposed theorem establishes stability conditions for the interval observer-based control (7) and it provides a constructive technique for $\underline{K}$ and $\bar{K}$ choice solving a series of LMIs. For $\bar{a}_{4}=\underline{a}_{4}=0$ the asymptotic stability is recovered.

\section{B. LPV case}

The assumptions (A1) and (A3) can be reformulated for (2) as follows.

There exists a matrix $\Delta A \geq 0$ such that

$$
A-\Delta A \leq A(\theta) \leq A+\Delta A \quad \forall \theta \in \Theta .
$$

Then for the system (2) the assumption (A3) is naturally satisfied for $\bar{a}_{1}=\bar{a}_{2}=\underline{a}_{1}=\underline{a}_{2}=|\Delta A|$ and $\bar{a}_{3}=\bar{a}_{4}=\underline{a}_{3}=$ $\underline{a}_{4}=0$. The problem of (3) stabilization in this case can be related with the Linear Differential Inclusion Control (LDIC) [8] (in general the proposed technique can be considered as a method to transform nonlinear output control problem to LDIC one). The result of Theorem 1 is also valid under (A4), therefore, in this section we concentrate on more simple situation assuming that $B(\theta)=B$, then $\underline{B}(u)=\bar{B}(u)=B$.

Applying the control (7) to the interval observer we get:

$$
\begin{aligned}
& \dot{\dot{x}}=[A-L C+B \underline{K}+\Delta A] \underline{x}+(B \bar{K}-\Delta A) \bar{x}+L y, \\
& \dot{\bar{x}}=[A-L C+B \overline{\bar{K}}+\Delta A] \bar{x}+(B \underline{K}-\Delta A) \underline{x}+L y .
\end{aligned}
$$

Introducing new variables $e_{d}=\bar{x}-\underline{x}$ (the estimated interval length, the difference between the upper and the lower estimates) and $e_{a}=0.5[\bar{x}+\underline{x}]$ (the interval average) we obtain for $K_{d}=0.5[\bar{K}-\underline{K}], K_{a}=\bar{K}+\underline{K}$ :

$$
\begin{aligned}
& \dot{e}_{d}=[A-L C+2 \Delta A] e_{d}, \\
& \dot{e}_{a}=\left[A-L C+B K_{a}\right] e_{a}+B K_{d} e_{d}+L y .
\end{aligned}
$$

In this case the dynamics of $e_{d}$ does not depend on the control and stability of this variable has to be ensured by a choice of the matrix $L$ (the pair $(A, C)$ is observable for instance). The choice $K_{d}=0$ may explicitly decouple the dynamics of the errors $e_{d}$ and $e_{a}$. This is a pure linear system whose internal stability is defined by the triangular matrix

$$
G=\left[\begin{array}{cc}
A-L C+2 \Delta A & 0 \\
B K_{d} & A-L C+B K_{a}
\end{array}\right],
$$

that is stable if the main diagonal blocks are Hurwitz.

Theorem 3. Let assumptions (A2) and (A4) hold, $\underline{x}(0) \leq$ $x(0) \leq \bar{x}(0)$ and there exist matrices $P^{T}=P \succ 0, Q^{T}=$ $Q \succ 0$ and $W$ such that the LMIs

$$
\begin{gathered}
{\left[\begin{array}{cc}
{\left[Q+|\Upsilon|^{2} \Lambda^{T} \Lambda\right]^{-1}} & P \\
P & \Pi-I
\end{array}\right] \succeq 0,} \\
\Pi=-P Z^{T}-Z P-W^{T} N^{T}-N W \\
Q+|\Upsilon|^{2} \Lambda^{T} \Lambda \succ 0, \Lambda=\left[0 L^{T}\right]^{T}, \Upsilon=[C C], \\
Z=\left[\begin{array}{cc}
A-L C+2 \Delta A & 0 \\
0 & A-L C
\end{array}\right], N=\left[\begin{array}{c}
0 \\
B
\end{array}\right]
\end{gathered}
$$

are satisfied. Then $\left[K_{d} K_{a}\right]=W P^{-1}$ and the system (2), (3), (7) is asymptotically stable.

Proof: All conditions of Theorem 1 are satisfied and $\underline{x}(t) \leq x(t) \leq \bar{x}(t) \forall t \geq 0$. Using the Schur complement the solution of the introduced LMIs implies that for $P^{T}=P \succ 0$, $Q^{T}=Q \succ 0$ and $W$ the Riccati inequality is satisfied:

$P Z^{T}+Z P+W^{T} N^{T}+N W+I+P\left[Q+|\Upsilon|^{2} \Lambda^{T} \Lambda\right] P \prec 0$.

Then for $\left[K_{d} K_{a}\right]=W P^{-1}$ the Riccati inequality is valid:

$$
G^{T} P^{-1}+P^{-1} G+P^{-2}+|\Upsilon|^{2} \Lambda^{T} \Lambda+Q \prec 0 .
$$

Consider the Lyapunov function $V=\varepsilon^{T} P^{-1} \varepsilon$ for $\varepsilon=$ $\left[e_{d}^{T} e_{a}^{T}\right]^{T}$, then

$$
\dot{V}=\varepsilon^{T}\left(G^{T} P^{-1}+P^{-1} G\right) \varepsilon+2 \varepsilon^{T} P^{-1} \Lambda y,|y| \leq|\Upsilon||\varepsilon|
$$

and $2 \varepsilon^{T} P^{-1} \Lambda y \leq \varepsilon^{T} P^{-2} \varepsilon+y \Lambda^{T} \Lambda y \leq \varepsilon^{T} P^{-2} \varepsilon+$ $|\Upsilon|^{2} \varepsilon^{T} \Lambda^{T} \Lambda \varepsilon$ since $y^{T} y \leq|\Upsilon|^{2} \varepsilon^{T} \varepsilon$. Thus we obtain:

$\dot{V} \leq \varepsilon^{T}\left(G^{T} P^{-1}+P^{-1} G+P^{-2}+|\Upsilon|^{2} \Lambda^{T} \Lambda\right) \varepsilon \leq-\varepsilon^{T} Q \varepsilon$. 

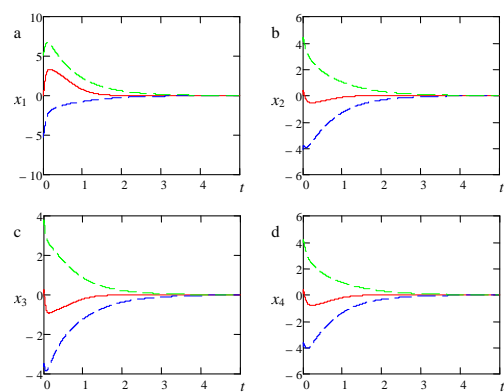

Figure 1. The state trajectories of LPV system.

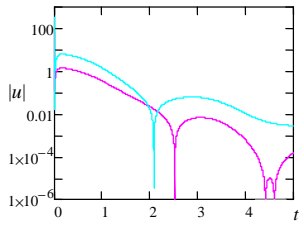

Figure 2. The control amplitudes for LPV system.

Therefore, the variable $\varepsilon$ is asymptotically stable, that implies the same property for the variable $x$.

Comparing with Theorem 2, Theorem 3 presents more simple way of the case $B(\theta)=B$ treatment.

\section{EXAMPLES}

In this section we illustrate the proposed approach on examples of time-varying uncertain systems.

\section{A. LPV system}

Consider the uncertain system:

$$
\begin{gathered}
\dot{x}=A(\theta) x+B u, y=x_{1}, \\
A-\Delta A \leq A(\theta) \leq A+\Delta A, \theta \in \mathbb{R}, \\
A=\left[\begin{array}{cccc}
35.6 & 50.7 & 45.6 & 75.6 \\
-1.8 & -25.5 & -3.8 & -6.3 \\
-18.1 & -20 & -38.9 & -31 \\
-5.8 & -7 & -6.6 & -30.5
\end{array}\right], \\
\Delta A=\frac{1}{4}\left[\begin{array}{cccc}
1 & 1 & 5 & 2 \\
1 & 2 & 1 & 3 \\
2 & 1 & 2 & 2 \\
1 & 4 & 1 & 2
\end{array}\right], B=\left[\begin{array}{cc}
1 & -1 \\
2 & 3 \\
-2 & 1 \\
-2 & 2
\end{array}\right],
\end{gathered}
$$

where the matrix $\Delta A$ defines the admissible time-varying deviations from the nominal value $A$ (assumption (A4) is true). For unstable matrix $A$ there is no $L$ such that $A-L C$ is Metzler (assumption (A2) fails to satisfy). However for

$$
\begin{gathered}
L=\left[\begin{array}{llll}
5 & 2 & 3 & 1
\end{array}\right]^{T}, \\
T=\left[\begin{array}{cccc}
-22.179 & 8 & 3 & 19 \\
8 & -21.179 & 7 & 5 \\
3 & 7 & -20.179 & 6 \\
9 & 5 & 6 & -19.179
\end{array}\right]
\end{gathered}
$$

the matrix $T^{-1}(A-L C) T$ is Hurwitz and Metzler as it is required in Remark 1 and Theorem 3. In addition, all other conditions of Theorem 3 are satisfied for the system (4) and the interval observer (5) with

$$
\begin{gathered}
\underline{K}=\left[\begin{array}{cccc}
-50.76 & -50.92 & -50.59 & -50.72 \\
267.17 & 267.91 & 266.30 & 26.08
\end{array}\right], \\
\bar{K}=\left[\begin{array}{cccc}
152.56 & 151.80 & 152.05 & 151.83 \\
-799.02 & -795.32 & -796.08 & -796.11
\end{array}\right],
\end{gathered}
$$

$$
P=10^{3}\left[\begin{array}{cccccccc}
1.60 & -0.32 & -0.67 & -0.41 & -0.97 & 0.07 & 0.39 & 0.31 \\
-0.32 & 0.81 & -0.15 & -0.26 & 0.13 & -0.06 & -0.13 & -0.01 \\
-0.67 & -0.15 & 1.01 & -0.08 & 0.50 & -0.15 & -0.35 & -0.08 \\
-0.41 & -0.26 & -0.08 & 0.52 & 0.24 & 0.09 & 0.04 & -0.14 \\
-0.97 & 0.13 & 0.50 & 0.24 & 1.93 & -0.44 & -0.77 & -0.63 \\
0.073 & -0.06 & -0.15 & 0.09 & -0.44 & 0.82 & -0.18 & -0.13 \\
0.39 & -0.13 & -0.35 & 0.04 & -0.77 & -0.18 & 0.97 & 0.03 \\
0.31 & -0.01 & -0.08 & -0.14 & -0.63 & -0.13 & 0.03 & 0.66
\end{array}\right]
$$

$Q=0.01 I$. The Robust Control Toolbox (MATLAB R2007b) was used to resolve the LMI. For simulation we choose $A[\theta(t)]=A(t)=A+V(t)$, where

$$
V(t)=\frac{1}{4}\left[\begin{array}{cccc}
\sin (t) & \cos (0.5 t) & 5 \sin (2 t) & 2 \cos (t) \\
\sin (0.5 t) & 2 \cos (2 t) & \cos (t) & 3 \sin (0.5 t) \\
2 \cos (2 t) & \sin (t) & 2 \cos (0.5 t) & 2 \sin (2 t) \\
\cos (t) & 4 \sin (0.5 t) & \cos (2 t) & 2 \sin (0.5 t)
\end{array}\right] .
$$

The results of simulation are presented in figures 1 and 2 . On plots Fig. 1,a - Fig. 1,d the state coordinates are shown (solid line) with the corresponding bounding variables from the interval observer (dashed lines). In Fig. 2 the controls amplitudes are given in the logarithmic scale.

\section{B. Nonlinear system}

Consider the time-varying nonlinear pendulum:

$$
\dot{x}_{1}=x_{2} ; \dot{x}_{2}=-\omega^{2}(t) \sin \left(x_{1}\right)-\kappa(t) x_{2}+b(t) u ; y=x_{1},
$$

where $x_{1} \in \mathbb{R}$ is the angle, $x_{2} \in \mathbb{R}$ is the angular velocity. The parameters satisfy the inequalities

$$
\begin{gathered}
0 \leq \omega_{m} \leq \omega(t) \leq \omega_{M}, \\
\kappa_{m} \leq \kappa(t) \leq \kappa_{M}, b_{m} \leq b(t) \leq b_{M}
\end{gathered}
$$

for some known $\omega_{m}, \omega_{M}, \kappa_{m}, \kappa_{M}, b_{m}, b_{M}$. Clearly, the system is in the form (1) for

$$
\begin{gathered}
A=\left[\begin{array}{cc}
0 & 1 \\
0 & -\kappa_{a}
\end{array}\right], B[\theta(t)]=\left[\begin{array}{c}
0 \\
\theta_{3}(t)
\end{array}\right], C=\left[\begin{array}{l}
1 \\
0
\end{array}\right]^{T}, \\
f\left[x_{1}, x_{2}, \theta(t)\right]=\left[\begin{array}{c}
0 \\
\theta_{1}(t) \sin \left(x_{1}\right)-\theta_{2}(t) x_{2}
\end{array}\right], \\
\theta(t)=\left[-\omega^{2}(t) \Delta \kappa(t) b(t)\right]^{T},
\end{gathered}
$$

where $\kappa_{a}=0.5\left(\kappa_{m}+\kappa_{M}\right), \Delta \kappa(t)=\kappa(t)-\kappa_{a},|\Delta \kappa(t)| \leq$ $\delta \kappa=0.5\left(\kappa_{M}-\kappa_{m}\right)$. Assumption (A1) is satisfied for

$\underline{f}[\underline{x}, \bar{x}, y]=\left[\begin{array}{cc}-\omega_{M}^{2} \sin (y) & \text { if } \sin (y)>0 \\ -\omega_{m}^{2} \sin (y) & \text { if } \sin (y) \leq 0\end{array}-\delta \kappa \max \left\{\left|\bar{x}_{2}\right|,\left|\underline{x}_{2}\right|\right\}\right]$,

$\bar{f}[\underline{x}, \bar{x}, y]=\left[\begin{array}{cc}-\omega_{M}^{2} \sin (y) & \text { if } \sin (y) \leq 0 \\ -\omega_{m}^{2} \sin (y) & \text { if } \sin (y)>0\end{array}+\delta \kappa \max \left\{\left|\bar{x}_{2}\right|,\left|\underline{x}_{2}\right|\right\}\right]$.

It is required to stabilize this pendulum in the upper unstable equilibrium $(\pi, 0)$ starting from the origin (the stable equilibrium). Such problem is called the pendulum swinging up, a solution of this problem for uncertain and time-varying 


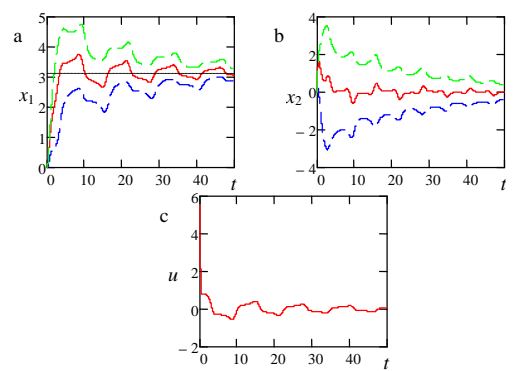

Figure 3. The results of nonlinear pendulum stabilization.

pendulum is rather complicated. For simulation the following values of parameters have been chosen:

$$
\begin{gathered}
\omega_{m}=0.1, \omega_{M}=1.2, \kappa_{m}=0.4, \kappa_{M}=1.5, \\
b_{m}=0.5, b_{M}=2.5 \\
\omega(t)=0.5\left[\omega_{M}+\omega_{m}+\left(\omega_{M}-\omega_{m}\right) \cos (t)\right], \\
b(t)=0.5\left[b_{M}+b_{m}+\left(b_{M}-b_{m}\right) \cos (2 t)\right], \\
\kappa(t)=\kappa_{a}+\delta \kappa \sin (t) .
\end{gathered}
$$

For $L=\left[\begin{array}{ll}2 & 0\end{array}\right]^{T}$ the matrix $A-L C$ becomes Hurwitz and Metzler (assumption (A2) is verified). Clearly, assumption (A3) holds since the nonlinearity $f$ is globally Lipschitz in this case and $\bar{a}_{1}=\underline{a}_{1}=\bar{a}_{2}=\underline{a}_{2}=\delta \kappa, \bar{a}_{4}=\underline{a}_{4}=0, \bar{a}_{3}=\underline{a}_{3}=\omega_{M}^{2}$, then $\alpha=4\left(2 \delta \kappa+\omega_{M}^{2}+|L|\right)=18.16$. The Robust Control Toolbox (MATLAB R2007b) used to resolve the LMIs from Theorem 2 indicated that for

$$
\begin{gathered}
\underline{K}=-[0.88211 .9851], \bar{K}=\underline{K}, Q=I, \epsilon=10^{4}, \\
P=10^{5}\left[\begin{array}{cccc}
0.6271 & 0.5224 & 0 & 0.0001 \\
0.5224 & 1.1758 & 0.0001 & 0.0002 \\
0 & 0.0001 & 0.6271 & 0.5224 \\
0.0001 & 0.0002 & 0.5224 & 1.1758
\end{array}\right]
\end{gathered}
$$

the LMIs are marginally feasible. The results of simulation are presented in Fig. 3, where in Fig. 3,a - Fig. 3,b the state coordinates (solid lines) and their interval bounds (dashed lines) are plotted, in Fig. 3,c the control is shown.

\section{CONCLUSIONS}

The problems of stabilization of nonlinear uncertain systems and LPV systems have been addressed. A new approach for output dynamic feedback design is proposed, where the conventional observer is replaced with an interval one. In this way, the problem of output stabilization of an uncertain nonlinear system is reduced to the problem of robust state feedback design for two completely known nonlinear systems. Applicability conditions of the interval observers are less conservative than for the conventional state observers. Thus the proposed approach extends the class of uncertain nonlinear systems having stabilizing control laws. Application of the proposed stabilizing method to LPV systems does not require the condition that the vector of scheduling parameters should be measured, making the method very attractive for uncertain systems with partial measurements. The price to pay is that the dimension of the controller may become rather large (the interval observer has the dimension twice bigger than the plant model). The proposed approach is illustrated through simulation examples.

Finally, the work presented in this paper is focused on the case of Lipschitz nonlinearities. Other state feedback techniques for robust stabilization of nonlinear systems (e.g. backstepping/forwarding, passivation approach, feedback linearization) can be applied in a similar way. An appealing direction for future work is to relax conservatism of the proposed LMI stability conditions.

\section{REFERENCES}

[1] P. Apkarian and P. Gahinet. A convex characterization of gain-scheduled h infinity controllers. IEEE Trans. Autom. Control, 40(5):853-864, 1995.

[2] A. Astolfi, D. Karagiannis, and R. Ortega. Non-linear and Adaptive Control with Applications. Communications and Control Engineering. Springer, 2008.

[3] P.-A. Bliman. Positive Polynomials in Control, volume 312 of Lecture Notes in Control and Information Sciences, chapter Stabilization of LPV Systems, pages 103-116. Springer, 2005.

[4] J. Daafouz, J. Bernussou, and J. C. Geromel. On inexact lpv control of continuous-time polytopic systems. IEEE Trans. Autom. Control, 53(7):1674-1678, 2008.

[5] J.L. Gouzé, A. Rapaport, and M.Z. Hadj-Sadok. Interval observers for uncertain biological systems. Ecological Modeling, 133:46-56, 2000.

[6] S. Hecker and A. Varga. Generalized lft-based representation of parametric uncertain models. European J. Control, 10(4):326-337, 2004.

[7] W. P. M. H. Heemels, J. Daafouz, and G. Millerioux. Observer-based control of discrete-time lpv systems with uncertain parameters. IEEE Trans. Autom. Control, 55(9):2130-2135, 2010.

[8] T Hu. Nonlinear control design for linear differential inclusions via convex hull of quadratics. Automatica, 43:685-692, 2007.

[9] A. Isidori. Nonlinear Control Systems. Springer Verlag, London, 3rd edition, 1995.

[10] L. Jetto and V. Orsini. Efficient lmi-based quadratic stabilization of interval lpv systems with noisy parameter measures. IEEE Trans. Autom. Control, 55(4):993-998, 2010.

[11] H.K. Khalil. Nonlinear Systems. Prentice Hall, Upper Saddle River, New Jersey, 3rd edition, 2002.

[12] I. E. Kose and F. Jabbari. Control of lpv systems with partly-measured parameters. IEEE Trans. Autom. Control, 44(3):658-663, 1999.

[13] G. Kreisselmeier and R. Engel. Nonlinear observers for autonomous lipschitz continuous systems. IEEE Trans. Autom. Control, 48(3):397401, 2003.

[14] L.H. Lee. Identification and Robust Control of Linear Parameter-Varying Systems. PhD thesis, Univ. of California at Berkeley, 1997.

[15] A. Marcos and J. Balas. Development of linear-parameter-varying models for aircraft. J. Guidance, Control, Dynamics, 27(2):218-228, 2004.

[16] F. Mazenc and O. Bernard. Asymptotically stable interval observers for planar systems with complex poles. IEEE Trans. Autom. Control, 55(2):523-527, 2010.

[17] A.M. Pertew, H.J. Marquez, and Q. Zhao. Hinf observer design for lipschitz nonlinear systems. IEEE Trans. Autom. Control, 51(7):12111216, 2006.

[18] A. Rapaport and J. Harmand. Robust regulation of a class of partially observed nonlinear continuous bioreactors. J. Process Control, 12:291302, 2002.

[19] T. Raïssi, D. Efimov, and A. Zolghadri. Interval state estimation for a class of nonlinear systems. IEEE Trans. Automatic Control, page to appear, January 2012

[20] T. Raïssi, G. Videau, and A. Zolghadri. Interval observers design for consistency checks of nonlinear continuous-time systems. Automatica, 46(3):518-527, 2010.

[21] W.J. Rugh and J.S. Shamma. Research on gain scheduling. Automatica, 36(10):1401-1425, 2000.

[22] J. Shamma and D. Xiong. Set-valued methods for linear parameter varying systems. Automatica, 35:1081-1089, 1999.

[23] H.L. Smith. Monotone Dynamical Systems: An Introduction to the Theory of Competitive and Cooperative Systems, volume 41 of Surveys and Monographs. AMS, Providence, 1995.

[24] W. Tan. Applications of Linear Parameter-Varying Control Theory. PhD thesis, University of California at Berkeley, 1997. 